\title{
Population study downgrades some copy number variants' impact on autism
}

\author{
BY CHARLES Q. CHOI
}

28 JANUARY 2022

\section{Listen to this story:}

https://www.spectrumnews.org/wpcontent/uploads/2022/01/audio-537a9431-0795-433b-84ca-6397d48b57cb-encodings.mp3

Some rare mutations linked to autism may boost a person's chances of having the condition less than prior work suggests, according to a new study, but they can also raise the likelihood of having other conditions.

Repeated or missing stretches of DNA, or copy number variations (CNVs), increase the chances of having any of multiple neurodevelopmental or psychiatric conditions, including autism. Previous studies, however, focused on people with clinical diagnoses. Because many people might carry a CNV without any obvious effects, it was unclear how much CNVs increased the chances of having a neuropsychiatric condition overall.

Now scientists have conducted what may be the first population-wide review of the prevalence and impact of CNVs in six chromosomal regions linked to a range of neurodevelopmental and psychiatric conditions: 1q21.1, 15q11.2, 15q13.3, 16p11.2, 17p12 and 17q12. They also looked for links between the CNVs and other conditions, such as epilepsy and fertility, and mortality.

Having a CNV in any of the regions except $17 \mathrm{p} 12$ significantly increased the likelihood of autism and most other diagnoses considered, including attention deficit hyperactivity disorder (ADHD), schizophrenia, major depressive disorder and intellectual disability, the results showed. And deletions in 1q21.1 substantially increased the risk of bipolar disorder in men and major depressive disorder in both sexes. CNVs were tied to physical ailments as well - a deletion in 15q13.3, for 


\section{Spectrum | Autism Research News}

https://www.spectrumnews.org

example, was linked to inflammation of the thyroid gland.

"We now have a realistic sense of which disorders can be expected and the likelihood that a given person develops a given illness," says lead investigator Thomas Werge, professor of clinical medicine at the University of Copenhagen in Denmark.

The results indicate that autistic people are more likely to carry CNVs than non-autistic people and stand to benefit from newborn screening, says Marianne van den Bree, professor of psychological medicine and clinical neurosciences at Cardiff University in Wales, who did not take part in this research.

"Ideally, the families of babies born with a copy number variant linked with high risk of a neuropsychiatric disorder should be offered the opportunity of continuous monitoring and early implementation of support," she says.

Werge and his colleagues analyzed genetic and health care data from 87,377 people born in Denmark between 1 May 1981 and 31 December 2005 who were included in the Lundbeck Foundation Initiative for Integrative Psychiatric Research (iPSYCH) database. The sample roughly 2 percent of the Danish population born during the period studied — included 57,377 people clinically diagnosed with ADHD, major depressive disorder, schizophrenia, autism or bipolar disorder, along with 30,000 people drawn at random from the database.

Two of the CNVs - deletions in 1q21.1 and 17q12 - boosted the likelihood of autism more than others, the team found. CNVs in 1q21.1, 15q13.3, 16p11.2 and 17q12 also increased the chances of having autism and co-occurring intellectual disability or ADHD - and, to a lesser extent, CNVs in $15 q 11.2$ and 17p12 did as well. The scientists detailed their results in November in JAMA Psychiatry.

Overall, the CNV-linked increases in risk were generally lower than previous estimates, which van den Bree says are likely upper estimates because they are based on analyses of clinically diagnosed groups.

Duplications in 16p11.2 increased the chances of autism in men, and deletions in this stretch increased the chances of autism in women. The risks that many CNVs pose to an individual may depend on sex, Werge says, which could explain a range of sex differences seen in psychiatric conditions.

Deletions in 17p12 lowered the likelihood of many of the psychiatric diagnoses, the researchers found. This discovery warrants follow up, they say, because if confirmed it could point toward new therapeutic opportunities.

Because this study followed people only until age 32, it does not provide insights into later-onset 


\section{Spectrum | Autism Research News}

https://www.spectrumnews.org

disorders, Werge cautions. And because the researchers relied on hospital records, they could not track clinical outcomes. They plan to follow socioeconomic status and non-hospital medical data, such as general-practitioner-prescribed medications in people with CNVs, in their next studies, Werge says. They also plan to study more CNVs, along with single-letter DNA variations.

Future research "should investigate the impact of these rare variants on early development," says Samuel Chawner, a research fellow in psychology at Cardiff University, who did not participate in this study. "However, this approach would require large-scale studies that conduct detailed developmental assessments that go beyond information available in medical records."

Scientists also need to study the implications of carrying CNVs in people of non-European ancestry, van den Bree says. "Most genetics research to date has been based on participants of European ancestry. It is poorly understood whether carrying a copy number variant has similar consequences in people of different ethnic backgrounds. Insights into this matter would allow better tailored care for patients and their families."

Cite this article: https://doi.org/10.53053/NPAH2204 